\author{
$2^{\prime \prime}-N$-FORMIMIDOYLSPORARICIN A \\ PRODUCED BY \\ SACCHAROPOLYSPORA \\ HIRSUTA SUBSP. KOBENSIS \\ Hamao Umezawa, Shuichi Gomi, \\ Yuji Yamagishi, TAMami Obata, \\ TAKaKo IKEDA, MASA HAMAdA \\ and SHINICHI KONDO \\ Institute of Microbial Chemistry, \\ 3-14-23 Kamiosaki, Shinagawa-ku, \\ Tokyo 141, Japan
}

(Received for publication June 3, 1986)

Sporaricin A, an aminoglycoside antibiotic containing a 1,4-diaminocyclitol was found by Deushi et al. ${ }^{1)}$ in the culture filtrate of Saccharopolyspora hirsuta subsp. kobensis. Related $N$-formimidoyl antibiotics, $2^{\prime \prime}-N$-formimidoylfortimicin A (dactimicin) ${ }^{2)}$ and $2^{\prime \prime}-N$-formimidoylistamycins $\mathrm{A}$ and $\mathrm{B}^{3)}$ were found by INOUYE et al. and by Kondo et al. in culture filtrates of Dactylosporangium matsuzakiense SF-2052 and Streptomyces tenjimariensis SS-939, respectively. In this paper, we will report the isolation and characterization of another related antibiotic, $2^{\prime \prime}-N$-formimidoylsporaricin A produced by the strain MG937-14F1. This strain was isolated from a soil sample collected at a garden of our Institute and classified into Saccharopolyspora hirsuta subsp. kobensis. ${ }^{4)}$ The production of $2^{\prime \prime}-N$-formimidoylsporaricin A by the sporaricinproducing strain has never been reported before.

Fermentation and Isolation

Strain MG937-14F1 was cultured at $30^{\circ} \mathrm{C}$ for

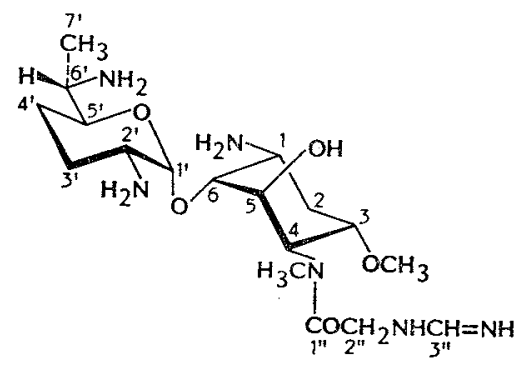

3 days on a rotatory shaker $(180 \mathrm{rpm})$ in a $500-$ $\mathrm{ml}$ baffled Erlenmeyer flask containing $110 \mathrm{ml}$ of a seed medium [dextrin $2 \%$, galactose $2 \%$, peptone (Bacto-Soytone) $1 \%$, corn steep liquor (Nisshoku) $0.5 \%,\left(\mathrm{NH}_{4}\right)_{2} \mathrm{SO}_{4} 0.2 \%$ and $\mathrm{CaCO}_{3}$ $0.2 \%, \mathrm{pH} 7.4]$. The seed culture $(2.2 \mathrm{ml})$ thus prepared was inoculated into $110 \mathrm{ml}$ of a production medium consisting of starch $3 \%$, soybean meal (Nisshin) $1.5 \%$, corn steep liquor $0.5 \%$, yeast extract (Oriental) $0.2 \%, \mathrm{NaCl} 0.3 \%, \mathrm{CaCO}_{3}$ $0.3 \%, \mathrm{MgSO}_{4} \cdot 7 \mathrm{H}_{2} \mathrm{O} 0.05 \%$ and $\mathrm{CoCl}_{2} \cdot 6 \mathrm{H}_{2} \mathrm{O}$ $0.001 \%(\mathrm{pH} 7.0)$ in a flask and cultured at $27^{\circ} \mathrm{C}$ for 4 days on a rotatory shaker.

The culture broth was harvested from 45 flasks and combined. It was suggested to contain $5.7 \mu \mathrm{g} / \mathrm{ml}$ of $2^{\prime \prime}-N$-formimidoylsporaricin $A$ by the cylinder plate test using Bacillus subtilis PCI219 as the test organism and pure $2^{\prime \prime}-\mathrm{N}$-formimidoylsporaricin A sulfate $(610 \mu \mathrm{g} /$

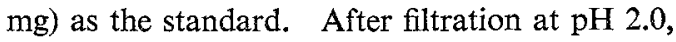
the antibiotic in the filtrate (adjusted to $\mathrm{pH} 7.0$, 4.03 liters) was adsorbed on a column of Amberlite IRC-50 $\left(70 \% \mathrm{Na}^{+}, 200 \mathrm{ml}\right)$ and eluted with $1 \mathrm{~N} \mathrm{HCl}$. The antibiotic in the active eluate (pH $7.2,230 \mathrm{ml}, 12.4 \mu \mathrm{g} / \mathrm{ml}$ ) was readsorbed on a column of charcoal $(2 \mathrm{~g})$ and eluted with $0.05 \mathrm{~N} \mathrm{HCl}$ in $80 \%$ aq methanol. The active eluate $(65 \mathrm{ml})$ was concd to dryness to give the crude powder (152 mg, $21.3 \mu \mathrm{g} / \mathrm{mg}$ ) in $14.1 \%$ yield from the filtrate. By HPLC analysis $^{5)}$ of components, the crude powder was shown to contain $2^{\prime \prime}-N$-formimidoylsporaricin A (32.84 minutes) at $1.6 \%$ and sporaricin A (21.49 minutes) at $0.3 \%$.

The crude powder was combined with other similar samples (91 $\mathrm{mg}$ of $63.9 \mu \mathrm{g} / \mathrm{mg}$ and $72 \mathrm{mg}$ of $21.4 \mu \mathrm{g} / \mathrm{mg})$ and dissolved in water $(10 \mathrm{ml}$, $1,059.3 \mu \mathrm{g} / \mathrm{ml})$. To the solution was added sodium $p$-toluenesulfonate $(200 \mathrm{mg})$ as the organic counter ion and the solution was adsorbed on a column of Diaion HP-20 $(10 \mathrm{ml})$. The column was developed successively with $50 \mathrm{ml}$ of water, each $50 \mathrm{ml}$, of $2.5 \%, 5.0 \%$, $7.5 \%, 10.0 \%$ and $12.5 \%$ aq methanol and $100 \mathrm{ml}$ of $15 \%$ aq methanol. The eluates were cut into 5-ml fractions. Fractions (Nos. 41 82, 260 $\mathrm{ml}, 24 \mu \mathrm{g} / \mathrm{ml}$ ) containing the antibiotic were combined and concd to $10 \mathrm{ml}$. Purification 
Table 1. 'H NMR chemical shifts of 2 "'-N-formimidoylsporaricin $\mathrm{A}(1)$ and sporaricin $\mathrm{A}$ (2) in $\mathrm{D}_{2} \mathrm{O}$ at $\mathrm{pD} 5.0$.

\begin{tabular}{|c|c|c|c|}
\hline \multirow{2}{*}{ Proton } & \multicolumn{2}{|c|}{1} & \multirow{2}{*}{$\stackrel{2}{\delta}(\mathrm{ppm})$} \\
\hline & $\delta(\mathrm{ppm})$ & Multiplicity & \\
\hline $1-\mathrm{H}$ & 3.92 & ddd & 3.90 \\
\hline $2-\mathrm{H}_{\mathrm{ax}}$ & 1.86 & ddd & 1.84 \\
\hline $2-\mathrm{H}_{\mathrm{eq}}$ & 2.66 & dddd & 2.63 \\
\hline $3-\mathrm{H}$ & 4.09 & ddd & 4.06 \\
\hline 4-H & 4.38 & $\mathrm{dd}$ & 4.36 \\
\hline $5-\mathrm{H}$ & 4.47 & $\mathrm{dd}$ & 4.43 \\
\hline $6-\mathrm{H}$ & 4.23 & br & 4.21 \\
\hline $3-\mathrm{OCH}_{3}$ & 3.48 & $\mathrm{~s}$ & 3.45 \\
\hline 4- $\mathrm{NCH}_{3}$ & 3.14 & s & 3.09 \\
\hline $1^{\prime}-\mathrm{H}$ & 5.51 & $\mathrm{~d}$ & 5.49 \\
\hline $2^{\prime}-\mathrm{H}$ & 3.64 & ddd & 3.63 \\
\hline $3^{\prime}-\mathrm{H}_{2}$ & $2.03 \sim 2.14$ & $\mathrm{~m}$ & $2.02 \sim 2.12$ \\
\hline $4^{\prime}-\mathrm{H}_{\mathrm{kx}}$ & 1.60 & $\mathrm{~m}$ & 1.58 \\
\hline $4^{\prime}-\mathrm{H}_{\mathrm{eq}}$ & $2.03 \sim 2.14$ & $\mathrm{~m}$ & $2.02 \sim 2.12$ \\
\hline $5^{\prime}-\mathrm{H}$ & 3.86 & ddd & 3.84 \\
\hline $6^{\prime}-\mathrm{H}$ & 3.39 & $\mathrm{dq}$ & 3.38 \\
\hline $7^{\prime}-\mathrm{H}_{3}$ & 1.35 & d & 1.33 \\
\hline $2^{\prime \prime}-\mathrm{H}_{\mathrm{a}}$ & 4.37 & $\mathrm{~d}$ & 4.03 \\
\hline $2^{\prime \prime}-\mathrm{H}_{\mathrm{b}}$ & 4.47 & d & 4.12 \\
\hline
\end{tabular}

$\delta(\mathrm{ppm})$ were measured from sodium 3-trimethylsilyl-1-propanesulfonate $(0 \mathrm{ppm})$ as the internal reference.
Table 2. ${ }^{13} \mathrm{C}$ NMR chemical shifts of $2^{\prime \prime}-N$ formimidoylsporaricin A (1) and sporaricin A (2) in $\mathrm{D}_{2} \mathrm{O}$ at $\mathrm{pD} 5.0$.

\begin{tabular}{|c|c|c|c|}
\hline \multirow{2}{*}{ Carbon } & \multicolumn{2}{|c|}{1} & \multirow{2}{*}{$\delta(\stackrel{2}{p p m})$} \\
\hline & $\delta(\mathrm{ppm})$ & Multiplicity & \\
\hline 1 & 47.5 & d & 47.3 \\
\hline 2 & 29.5 & $\mathrm{t}$ & 29.5 \\
\hline 3 & 72.2 & $\mathrm{~d}$ & 72.0 \\
\hline 4 & 56.7 & $\mathrm{~d}$ & 56.7 \\
\hline 5 & 68.1 & $\mathrm{~d}$ & 68.1 \\
\hline 6 & 73.1 & $\mathrm{~d}$ & 73.2 \\
\hline $3-\mathrm{OCH}_{3}$ & 56.7 & $\mathrm{q}$ & 56.6 \\
\hline 4- $\mathrm{NCH}_{3}$ & 32.1 & $\mathrm{q}$ & 32.1 \\
\hline $1^{\prime}$ & 92.6 & $\mathrm{~d}$ & 92.6 \\
\hline $2^{\prime}$ & 49.8 & $\mathrm{~d}$ & 49.7 \\
\hline $3^{\prime}$ & 21.5 & $\mathrm{t}$ & 21.4 \\
\hline $4^{\prime}$ & 26.4 & $\mathrm{t}$ & 26.5 \\
\hline $5^{\prime}$ & 71.2 & d & 71.0 \\
\hline $6^{\prime}$ & 52.0 & $\mathrm{~d}$ & 51.9 \\
\hline $7^{\prime}$ & 15.2 & $q$ & 15.3 \\
\hline $1^{\prime \prime}$ & 169.2 & s & 168.4 \\
\hline $2^{\prime \prime}$ & 44.3 & $\mathrm{t}$ & 41.4 \\
\hline $3^{\prime \prime}$ & 156.1 & $\mathrm{~d}$ & \\
\hline
\end{tabular}

Table 3. Minimum inhibitory concentrations of $2^{\prime \prime}-N$-formimidoylsporaricin A.

\begin{tabular}{|c|c|c|c|}
\hline Test organism & $\mu \mathrm{g} / \mathrm{ml}$ & Test organism & $\mu \mathrm{g} / \mathrm{ml}$ \\
\hline Staphylococcus aureus FDA 209P & 0.78 & Klebsiella pneumoniae PCI602 & 1.56 \\
\hline S. aureus Smith & 0.39 & K. pneumoniae $22 ¥ 3038$ & 3.13 \\
\hline S. aureus Ap01 & 6.25 & Shigella dysenteriae JS11910 & 3.13 \\
\hline S. epidermidis 109 & 0.78 & S. flexneri 4 b JS11811 & 3.13 \\
\hline Micrococcus flavus FDA16 & 1.56 & S. sonnei JS11746 & 3.13 \\
\hline M. luteus PCI1001 & 0.78 & Salmonella typhi T-63 & 0.78 \\
\hline Bacillus anthracis & 0.78 & S. enteritidis 1891 & 3.13 \\
\hline B. subtilis PCI 219 & 0.78 & Proteus vulgaris OX19 & 1.56 \\
\hline B. subtilis NRRL B-558 & 0.39 & $P$. rettgeri GN311 & 0.78 \\
\hline B. cereus ATCC10702 & 6.25 & P. rettgeri GN466 & 0.78 \\
\hline Corynebacterium bovis 1810 & 0.39 & Serratia marcescens & 3.13 \\
\hline Mycobacterium smegmatis ATCC607 & 0.78 & Serratia sp. SOU & 50 \\
\hline Escherichia coli NIHJ & 0.78 & Serratia sp. 4 & 25 \\
\hline E. coli $\mathrm{K}-12$ & 0.78 & Providencia sp. Pv16 & 1.56 \\
\hline E. coli $\mathrm{K}-12 \mathrm{R} 5$ & 3.13 & Providencia sp. 2991 & 1.56 \\
\hline E. coli $\mathrm{K}-12 \mathrm{R} 388$ & 0.78 & Pseudomonas aeruginosa A3 & 1.56 \\
\hline E. coli $\mathrm{K}-12$ J5R $11-2$ & 0.78 & $P$. aeruginosa No. 12 & 25 \\
\hline E. coli $\mathrm{K}-12 \mathrm{ML} 1629$ & 1.56 & P. aeruginosa $\mathrm{H} 9$ & 25 \\
\hline E. coli $\mathrm{K}-12$ ML1630 & 1.56 & $P$. aeruginosa $\mathrm{H} 11$ & 50 \\
\hline E. coli $\mathrm{K}-12 \mathrm{ML} 1410$ & 1.56 & $P$. aeruginosa TI-13 & 25 \\
\hline E. coli $\mathrm{K}-12$ ML1410 R81 & 1.56 & $P$. aeruginosa $\mathrm{GN} 315$ & 25 \\
\hline E. coli $\mathrm{K}-12$ LA290 R55 & 1.56 & P. aeruginosa 99 & $>100$ \\
\hline E. coli $\mathrm{K}-12 \mathrm{LA} 290 \mathrm{R} 56$ & 1.56 & $P$. aeruginosa $\mathrm{B}-13$ & $>100$ \\
\hline E. coli K-12 LA290 R64 & 1.56 & $P$. aeruginosa $21-75$ & 50 \\
\hline E. coli $\mathrm{W} 677$ & 0.78 & P. aeruginosa PSTI & 50 \\
\hline E. coli JR66/W677 & 0.78 & P. aeruginosa ROS134/PU21 & $>100$ \\
\hline E. coli $\mathrm{K}-12$ C600 R 135 & 25 & $P$. aeruginosa $\mathrm{K}-\mathrm{Ps} 102$ & 50 \\
\hline E. coli JR225 & 0.78 & P. maltophilia GN907 & $>100$ \\
\hline
\end{tabular}


of the antibiotic in the concentrate was achieved by Amberlite CG-50 $\left(70 \% \mathrm{Na}^{+}, 6 \mathrm{ml}\right)$ column chromatography eluted with $0.5 \mathrm{~N} \mathrm{H}_{2} \mathrm{SO}_{4}$ followed by charcoal $(500 \mathrm{mg})$ column chromatography eluted with $0.01 \mathrm{~N}_{2} \mathrm{SO}_{4}$. After neutralizing the eluate with Amberlite IR-45 $\left(\mathrm{OH}^{-}\right)$ and concentrating to $4 \mathrm{ml}$, the concentrate was passed through a column of Amberlite IRA-400 $\left(\mathrm{SO}_{4}{ }^{2-}, 4 \mathrm{ml}\right)$ and developed with water. The active effluent $(4 \mathrm{ml})$ was lyophilized to give 7.6 mg of pure 2 " $-N$-formimidoylsporaricin $\mathrm{A}$ disulfate tetrahydrate $(610 \mu \mathrm{g} / \mathrm{mg})$ as a colorless hygroscopic powder in $43.8 \%$ yield from the crude powder.

\section{Properties and Structure}

$2^{\prime \prime}-N$-Formimidoylsporaricin A disulfate tetrahydrate: No definite $\mathrm{mp}[\alpha]_{\mathrm{D}}^{27}+65.8^{\circ}$ (c) 0.5 , $\mathrm{H}_{2} \mathrm{O}$ ), secondary ion mass spectrum (SI-MS) $\mathrm{m} / \mathrm{z}$ $417\left(\mathrm{MH}^{+}\right)$, IR $(\mathrm{KBr}) \mathrm{cm}^{-1} 3400,2950,1710$ $\left(\mathrm{C}=\mathrm{N}^{+}\right), 1640,1490,1120,1050,990$. Anal Calcd for $\mathrm{C}_{18} \mathrm{H}_{30} \mathrm{~N}_{8} \mathrm{O}_{5} \cdot 2 \mathrm{H}_{2} \mathrm{SO}_{4} \cdot 4 \mathrm{H}_{2} \mathrm{O}: \mathrm{C} 31.57$, $\mathrm{H}$ 7.07, N 12.27, $\mathrm{S}$ 9.36. Found: $\mathrm{C} 31.37, \mathrm{H}$ 6.48, N 11.47, S 9.20. By high-voltage paper electrophoresis with $3,500 \mathrm{~V}$ for 15 minutes in $\mathrm{HCOOH}-\mathrm{CH}_{3} \mathrm{COOH}-\mathrm{H}_{2} \mathrm{O}(1: 3: 36)$, the antibiotic moved toward the cathode with $\mathrm{Rm}$ (relative mobility to alanine) 2.01 . The ${ }^{1} \mathrm{H}$ and ${ }^{13} \mathrm{C}$ NMR chemical shifts in $\mathrm{D}_{2} \mathrm{O}$ (pD 5.0) were compared with those of sporaricin $A^{\theta)}$ as shown in Tables 1 and 2 , respectively. The ${ }^{13} \mathrm{C}$ NMR data of the antibiotic showed good agreements with those of sporaricin A except the $2^{\prime \prime}-\mathrm{C}$ and $3^{\prime \prime}-\mathrm{C}$ signals as shown in Table 2. These two signals were easily assigned from those (44.1 and $155.8 \mathrm{ppm}$ ) of $2^{\prime \prime}-N$-formimidoylistamycin B. ${ }^{5)}$ Therefore, the structure of the antibiotic was determined to be $2^{\prime \prime}-N$-formimidoylsporari- $\operatorname{cin} \mathrm{A}$.

As shown in Table 3, the antibiotic had strong antibacterial activity against Gram-positive and Gram-negative bacteria including aminoglycoside-resistant bacteria.

\section{References}

1) Deushi, T.; A. IwaSAKr, K. KamiYA, T. KuniedA, T. Mizoguchi, M. Nakayama, H. IтоH, T. MORI \& T. ODA: A new broad-spectrum aminoglycoside antibiotic complex, sporaricin. I. Fermentation, isolation and characterization. J. Antibiotics 32: $173 \sim 179,1979$

2) Inouye, S.; K. Ohba, T. Shomura, M. Kojima, T. Tsuruoka, J. Yoshida, N. Katö, M. Itō, S. Amano, S. OMoto, N. Ezaki, T. Itō, T. NiIDA \& K. WATANABE: A novel aminoglycoside antibiotic, substance SF-2052. J. Antibiotics 32: $1354 \sim 1356,1979$

3) Kondo, S.; Y. HoriuchI, D. IKedA, S. GoMI, K. HotTA, Y. OKAMI \& H. UMEZAWA: $2^{\prime \prime}-N$ Formimidoylistamycin A and $\mathbf{B}$ produced by Streptomyces tenjimariensis. J. Antibiotics 35: $1104 \sim 1106,1982$

4) IwasaKi, A.; H. ItoH \& T. MoRi: A new broad-spectrum aminoglycoside antibiotic complex, sporaricin. II. Taxonomic studies on the sporaricin producing strain Saccharopolyspora hirsuta subsp. kobensis nov. subsp. J. Antibiotics 32: $180 \sim 186,1979$

5) HorIuchI, Y.; D. IKEDA, S. Gom, S. Kondo \& H. UMEZAWA: Isolation and synthesis of $2^{\prime \prime}-$ $N$-formimidoylistamycins $\mathrm{A}$ and $\mathrm{B}$, new istamycin components. Carbohydr. Res. 109: 25 31, 1982

6) Deushi, T.; M. Nakayama, I. Watanabe, T. MOR, H. NaGANAwa \& H. UMEZAWA: A new broad-spectrum aminoglycoside antibiotic complex, sporaricin. III. The structure of sporaricins A and B. J. Antibiotics 32: 187 192, 1979 